\title{
Oclusão Percutânea dos Defeitos do Septo Atrial Utilizando Mais de Uma Prótese
}

\author{
Francisco Chamié ${ }^{1,2,3}$, Daniel Chamié ${ }^{4}$, Sérgio Ramos ${ }^{2,3}$, \\ João Carlos Tress ${ }^{5}$, Rosaura Victer ${ }^{5}$
}

\section{RESUMO}

Introdução: O fechamento percutâneo das comunicações interatriais (CIAs) é uma alternativa à cirurgia, aceita em todo o mundo. $\mathrm{O}$ uso de mais de uma prótese tem sido descrito, mas ainda há poucos estudos sobre o assunto, especialmente no que diz respeito ao seguimento a longo prazo. Neste trabalho, apresentamos nossa experiência com o uso de mais de uma prótese para o fechamento dos defeitos atriais múltiplos, e discutimos as diferentes condições em que isso se faz necessário. Método: Este é um estudo retrospectivo, em que foram analisados todos os casos submetidos a fechamento percutâneo de CIAs, no período de abril de 1999 a dezembro de 2007. Do total de casos, selecionamos aqueles portadores de defeitos múltiplos, concentrando nosso relato nos que necessitaram de mais de uma prótese para sua oclusão. Incluímos, também, dois casos de pacientes com shunt residual após o implante prévio de uma primeira prótese e que necessitaram de um segundo dispositivo. Os casos foram todos selecionados por meio de ecocardiogramas transesofágicos (ETE). Resultados: No período mencionado, 211 pacientes foram submetidos a fechamento percutâneo de CIAs. Em $12(5,6 \%)$ desses pacientes foi preciso utilizar duas próteses para oclusão de seus defeitos, como se segue: oito pacientes tinham dois orifícios; dois tinham três orifícios; e dois tinham quatro orifícios. A média das idades foi de $27,5 \pm 14,6$ anos. Dois casos apresentaram acidentes isquêmicos cerebrais prévios. Foram utilizadas 24 próteses, sendo 23 Amplatzer para CIAs e uma Amplatzer para forame oval (FOP). O implante foi possível em todos os casos. O diâmetro dos orifícios menores era de 10,5 \pm 4,0 mm e dos maiores, de 20,6 $\pm 5,5 \mathrm{~mm}$. O diâmetro central das próteses de CIA variou de $5 \mathrm{~mm}$ a $34 \mathrm{~mm}(16,8 \pm 7,6 \mathrm{~mm})$. Não houve complicações graves ou óbitos. Shunt residual imediato estava presente em dois $(16,6 \%)$ pacientes. Foram

\section{SUMMARY}

Transcatheter Occlusion of Atrial Septal Defects Using More Than One Device

Introduction: Transcatheter Atrial Septal Defect (ASD) closure is a worldwide accepted alternative to surgical repair. The use of more than one device has been described, however, there are just a limited number of studies available with a long term follow-up. In this study, the authors present their experience in transcatheter closure of multiple atrial septal defects utilizing more than one device, and discuss the different situations where this approach has become necessary. Methods: This is a retrospective study where all patients submitted to ASD device closure from April 1999 to December 2007 were analyzed. We selected those with multiple ASD and focused our study on those who had two devices implanted. We also included two patients who had significant residual shunts after the first procedure and were closed with a second device. All patients were selected based on transesophageal echocardiogram (TEE) results. Results: In the aforementioned period, 211 patients were submitted to transcatheter ASD closure. Twelve $(5.6 \%)$ patients needed two devices to close their defects, described as follows: eight patients had 2 orifices, two had 3 orifices, and two had 4 orifices. Mean age was $27.5 \pm 14.6$ years. Two patients suffered ischemic cerebral events (stroke) prior to occlusion. Twentyfour devices were utilized: 23 Amplatzer ASD Occluder devices and one $25 \mathrm{~mm}$ Amplatzer PFO Occluder device. The device implantation was possible in all cases. Average diameter for small defects was $10.5 \pm 4.0 \mathrm{~mm}$, and 20.6 $\pm 5.5 \mathrm{~mm}$ for larger defects. Mean central diameter of the devices varied from 5 to $34 \mathrm{~mm}(16.8 \pm 7.6 \mathrm{~mm})$. There were no major complications or deaths related to the procedure. Immediate post-procedural residual shunts were

\footnotetext{
1 Hospital dos Servidores do Estado - MS-RJ - Rio de Janeiro, RJ.

2 CARPE - Cardiologia Pediátrica e Fetal - Rio de Janeiro, RJ.

3 INTERCAT - Cardiologia Intervencionista - Rio de Janeiro, RJ.

4 Instituto Dante Pazzanese de Cardiologia - São Paulo, SP.

5 Hospital de Clínicas de Niterói - Niterói, RJ.

Correspondência: Francisco Chamié. Rua Dois de Dezembro, 38 -

sala 807 - Flamengo - Rio de Janeiro, RJ - CEP 22220-040.

Tel.: (21) 2246-1629 - Fax: (21) 2556-6833

E-mail: fchamie@pobox.com

Recebido em: 25/12/2007 • Aceito em: 21/1/2008
} 
acrescentados à casuística mais dois casos de shunt residual significativo, fechados, com sucesso, com uma segunda prótese Amplatzer para CIA. Conclusões: Apesar de tecnicamente mais complexo, o fechamento das CIAs com duas próteses é possível, seguro e eficaz. Até o presente momento, o uso de duas próteses metálicas não parece ser danoso ao septo atrial ou a estruturas vizinhas. Apesar disso, mais estudos são necessários para definir a segurança desse procedimento, especialmente em crianças.

DESCRITORES: Defeitos do septo interatrial. Cateterismo cardíaco. Próteses e implantes. present in two $(16.6 \%)$ patients. Two additional patients were included with significant residual shunt that required a second Amplatzer ASD device. Conclusion: Although technically more demanding, two device ASD closure is feasible, safe and effective. Implanting two metallic devices does not seem to be deleterious to atrial septum or surrounding structures, so far. In spite of that, more studies are deemed necessary to define the safety of the procedure, especially in children.
A s comunicações interatriais (CIAs) representam cerca de $10 \%$ das anomalias cardíacas congênitas. Aproximadamente 7,3\% dos portadores de ClA têm defeitos múltiplos ${ }^{1}$. Mais ou menos 2,2\% são portadores de aneurisma de septo atrial, com ou sem múltiplas perfurações ${ }^{2}$.

O fechamento dos defeitos com grande shunt esquerda-direita (E-D) é necessário para prevenir insuficiência cardíaca direita, hiper-resistência vascular pulmonar, arritmias atriais e, ainda, embolias paradoxais ${ }^{3}$.

O fechamento percutâneo das CIAs se tornou o método de escolha na maioria dos serviços em todo o mundo.

Ocasionalmente, dependendo da distância entre os orifícios, mais de uma prótese pode se fazer necessária para a completa oclusão dos defeitos ${ }^{4}$.

No presente trabalho, são discutidas as indicações para o uso de mais de uma prótese para fechamento das CIAs, os aspectos técnicos relativos ao procedimento, bem como suas potenciais complicações.

\section{MÉTODO}

\section{Desenho do estudo}

Este estudo apresenta uma análise retrospectiva, em que foram revisados os registros de todos os pacientes portadores de CIA submetidos a fechamento percutâneo com prótese, no período de abril de 1999 a dezembro de 2007. Foram identificados os pacientes portadores de CIAs múltiplas em quem foi necessário o implante de mais de uma prótese para o fechamento de seus defeitos.

\section{Seleção dos pacientes}

No período mencionado, todos os pacientes portadores de $\mathrm{ClA}$, com mais de cinco anos de vida ou $15 \mathrm{~kg}$ de peso, foram, consecutivamente, submetidos
DESCRIPTORS: Heart septal defects, atrial. Heart catheterization. Prostheses and implants.

a avaliação para fechamento percutâneo em nosso Serviço. A avaliação foi feita por meio da realização de ecocardiogramas transesofágicos (ETE). Todos os pacientes com múltiplos orifícios tratados com implante de mais de uma prótese foram considerados para análise. Selecionamos, também, os casos em que foi necessário o uso de uma segunda prótese para o fechamento de shunts residuais hemodinamicamente significativos.

\section{Prótese}

Os dispositivos de oclusão utilizados neste trabaIho foram as próteses Amplatzer. Em todos os casos, foi implantada prótese Amplatzer para CIA (Amplatzer Septal Occluder - AGA Medical Corp., Golden Valley, Minnesota, Estados Unidos), exceto em um, em que foi utilizada uma prótese Amplatzer para forame oval (FOP) (Amplatzer PFO Occluder - AGA Medical Corp., Golden Valley, Minnesota, Estados Unidos). Ambos os dispositivos já foram descritos, e suas características e técnica de implante já foram abordadas em publicações anteriores ${ }^{5,6}$.

\section{Ecocardiograma transesofágico}

Além da ótima visualização do defeito, da mensuração de seu diâmetro estático e da análise de fluxos, foi examinada, rotineiramente, toda a extensão do septo atrial, visando à detecção de mais de um orifício ou da presença de aneurisma.

Nos casos de defeitos múltiplos, realizava-se a mensuração do diâmetro estático de cada um, da distância entre eles, e da proximidade com outras estruturas intracardíacas de relevância.

Aneurisma do septo atrial (ASA) era definido, de acordo com os critérios de Mügge et al. ${ }^{7}$, como deslocamento do septo atrial de mais de $10 \mathrm{~mm}$ da linha média, na direção do átrio tanto direito como esquerdo. Didaticamente, consideramos como tipo A quando 
o deslocamento era apenas para o átrio direito, tipo B quando era apenas para o átrio esquerdo, e tipo C quando o movimento se fazia para ambos os átrios. Quando a movimentação do septo atrial não preenchia esse critério, ele era considerado, apenas, redundante.

\section{Técnica do procedimento}

Todos os procedimentos foram realizados sob anestesia geral, com intubação orotraqueal, e monitorados por ETE.

O acesso vascular era, preferencialmente, realizado por punção da veia femoral direita. Ocasionalmente, foram necessárias punções adicionais no mesmo vaso ou no vaso contralateral para a colocação da segunda prótese.

Os pacientes receberam heparinização sistêmica na dose de $100 \mathrm{UI} / \mathrm{kg}$ ou $5.000 \mathrm{UI}$ em adultos. Se o procedimento durasse mais de uma hora, era dada metade da dose original, a cada 30 minutos, até o término do exame.

Era administrada, também, cefalotina venosa (50 $\mathrm{mg} / \mathrm{kg}$, máximo $2 \mathrm{~g}$ ) no ato operatório, seguida por mais duas doses de cefalexina oral (25 mg/kg ou $500 \mathrm{mg}$ ) para profilaxia antimicrobiana após o procedimento.

Com o auxílio de visualização ecocardiográfica, era tomado sempre o cuidado de cruzar o septo atrial pelo orifício maior ou pelo mais central. Em seguida, era introduzido balão medidor AGA (AGA Medical Corporation - Golden Valley, Minnesota, Estados Unidos) de $24 \mathrm{~mm}$ ou $34 \mathrm{~mm}$, conforme a medida do diâmetro estático do defeito. O balão era insuflado até a completa oclusão do defeito (stop-flow technique), realizando a medida do seu diâmetro estirado. Os resultados obtidos pelo ETE e pela fluoroscopia eram comparados, sendo escolhido o mais adequado. Ao mesmo tempo, era verificada, também, a persistência de fluxo residual e a oclusão dos outros orifícios.

Quando os defeitos eram próximos, em geral confluíam com um orifício único ou a compressão do balão acabava por abolir completamente o shunt pelos outros orifícios. Nesse caso, era implantada uma única prótese, segundo a técnica descrita anteriormente ${ }^{8}$.

Nos casos em que os orifícios adicionais estavam muito afastados do orifício maior (geralmente acima de $8 \mathrm{~mm}$ ), era realizada nova punção da mesma veia femoral ou da veia contralateral e introduzido outro cateter, cruzando o defeito remanescente. Através dele era introduzido, sobre guia, novo balão medidor, sendo obtido o diâmetro estirado do novo defeito.

O septo interatrial era novamente avaliado, com Doppler colorido, para verificar a presença de shunt residual, classificado segundo Boutin et al. ${ }^{9}$ em: míni$\mathrm{mo}$, se $\leq 1 \mathrm{~mm}$; pequeno, se $\leq 2 \mathrm{~mm}$; moderado, se $>2 \mathrm{~mm}$ e $\leq 4 \mathrm{~mm}$; e grande, se $\geq 4 \mathrm{~mm}$.
Na ausência de shunts residuais, eram introduzidas próteses do mesmo tamanho ou $1 \mathrm{~mm}$ a $2 \mathrm{~mm}$ maiores que o diâmetro estirado de cada orifício.

Inicialmente, era implantada a prótese menor, que era deixada ainda presa ao sistema liberador. Em seguida, era implantada a maior, de forma a fazer com que os discos envolvessem a menor. Se a fluoroscopia e o ETE mostrassem boa estabilidade dos dispositivos e ausência de shunt residual, as próteses eram liberadas em seqüência, primeiro a menor e depois a maior.

Se houvesse oclusão temporária de todos os defeitos durante a presença do balão no orifício maior, mas após a colocação da prótese voltasse a haver fluxo pelo orifício menor, era tentado o implante de outra prótese, cruzando o septo pelo orifício menor, conforme já descrito. Nesses casos, a prótese maior não necessariamente envolvia a menor e o implante da segunda era feito após a primeira estar completamente liberada. Em alguns casos, as duas próteses ficavam completamente afastadas ou, mesmo, em planos diferentes uma da outra.

Nos pacientes que apresentaram shunt residual, pelo menos pequeno, após um ano, o defeito foi cruzado com o auxílio de guia hidrofílica e realizada a medida do diâmetro estirado. O balão medidor, ao ser insuflado, ajudou também na delineação da forma do pertuito a ser fechado. Em seguida, era escolhida a prótese mais adequada ao caso e implantada segundo a técnica habitual.

\section{Análise estatística}

$\mathrm{Na}$ análise estatística descritiva, as variáveis contínuas são expressas como média e desvio padrão e as variáveis categóricas, como números e porcentagens.

\section{RESULTADOS}

De abril de 1999 a dezembro de 2007, foram submetidos a fechamento percutâneo das CIAs 211 pacientes, dos quais $12(5,6 \%)$ apresentaram orifícios múltiplos, necessitando duas próteses.

A Tabela 1 apresenta as características demográficas e hemodinâmicas dos pacientes tratados com duas próteses. A distribuição por sexo mostrou oito pacientes do sexo feminino e quatro, do masculino. As idades variaram entre 8 anos e 51 anos $(27,5 \pm 14,6$ anos) e os pesos, de $25 \mathrm{~kg}$ a $109 \mathrm{~kg}(58,8 \pm 25,7 \mathrm{~kg})$.

Um paciente era portador de síndrome de Down (FISD, 17 anos). A indicação para o fechamento foi a presença de sobrecarga volumétrica das câmaras direitas, em dez pacientes. Os outros dois pacientes tiveram seus defeitos diagnosticados por apresentarem quadros neurológicos prévios, que foram a razão do encaminhamento: um deles apresentava duas CIAs pequenas (MCMV, 34 anos) e o outro, um FOP com pequena $\mathrm{CIA}$ adicional (COW, 51 anos). 
TABELA 1

Características demográficas e hemodinâmicas dos pacientes que receberam duas próteses $(\mathrm{n}=14)$

\begin{tabular}{lccccccc}
\hline $\mathbf{N}$ & ID & Sexo & $\begin{array}{c}\text { Idade } \\
\text { (anos) }\end{array}$ & $\begin{array}{c}\text { Peso } \\
(\mathbf{k g})\end{array}$ & $\begin{array}{c}\text { PAP } \\
(\mathbf{m m H g})\end{array}$ & $\begin{array}{c}\varnothing \text { estático** } \\
(\mathbf{m m})\end{array}$ & $\begin{array}{c}\varnothing \text { estirado** } \\
(\mathbf{m m})\end{array}$ \\
\hline 1 & FISD & $\mathrm{M}$ & 17 & 45 & $40 / 19(27)$ & $4+10+10$ & $13+26$ \\
2 & FCC & $\mathrm{F}$ & 45 & 65 & $26 / 12(18)$ & $4+15$ & $11+20$ \\
3 & AMA & $\mathrm{F}$ & 41 & 109 & $19 / 12(27)$ & $3+4+6+19$ & $4+24$ \\
4 & SAS & $\mathrm{M}$ & 13 & 30 & $26 / 10(18)$ & $3+16$ & $12+28$ \\
5 & DFSC & $\mathrm{F}$ & 21 & 40 & $35 / 17(26)$ & $10+27$ & $12+27$ \\
6 & SDM & $\mathrm{F}$ & 17 & 50 & $37 / 12(26)$ & $8+16$ & $14+21$ \\
7 & AMM & $\mathrm{F}$ & 9 & 25 & $22 / 11(17)$ & $2,5+3+4,5$ & $9+16$ \\
8 & MSA & $\mathrm{M}$ & 8 & 28 & $23 / 2(15)$ & $3+6$ & $10+14$ \\
9 & MCMV & $\mathrm{F}$ & 34 & 80 & $15 / 9(12)$ & $5+8$ & $15+16$ \\
10 & COW & $\mathrm{F}$ & 51 & 100 & $30 / 19(22)$ & $\mathrm{FO}+?$ & $4+11$ \\
11 & ALS & $\mathrm{F}$ & 21 & 40 & $18 / 11(14)$ & $3+8$ & $16+25$ \\
12 & ESG & $\mathrm{M}$ & 35 & 91 & $33 / 18(25)$ & $(2+3)(3+6)$ & $6+20$ \\
13 & GBV* & $\mathrm{F}$ & 7 & 22 & $26 / 16(21)$ & 4 & 8,5 \\
14 & SCS* & $\mathrm{F}$ & 41 & 70 & $30 / 13(19)$ & 8 & 18 \\
\hline
\end{tabular}

* Pacientes incluídos por apresentarem shunt residual após implante prévio de uma prótese. ** Os diâmetros estáticos representam o diâmetro medido de cada orifício individual e os diâmetros estirados, os orifícios resultantes após a medida com o balão. $\mathrm{N}=$ número do paciente; ID = identificação do paciente; PAP = pressão arterial pulmonar.

Dos 12 pacientes que estavam sendo submetidos ao procedimento pela primeira vez, o ETE foi capaz de detectar os orifícios adicionais em dez (83,3\%) e o exame já foi planejado contando com a possibilidade de uma segunda prótese. Em dois $(16,6 \%)$ casos, os defeitos menores foram reconhecidos por terem sido cruzados inadvertidamente com a guia. Em um deles, pela sua localização, o ETE não conseguiu acompanhar o implante da prótese, o que foi feito exclusivamente pela fluoroscopia. Em função disso, o planejamento do exame teve que ser alterado para a colocação de um segundo dispositivo.

Quanto ao número de orifícios, oito pacientes tinham dois orifícios, dois pacientes tinham três orifícios, e dois tinham quatro orifícios (Figura 1). Apenas cinco pacientes apresentaram aneurismas: dois do tipo A e três do tipo C. Os aneurismas tipo A tinham dois orifícios cada e os três do tipo $\mathrm{C}$ apresentavam, respectivamente, dois, três e quatro orifícios. Num dos casos, a paciente $(\mathrm{COW})$ tinha um aneurisma do tipo $\mathrm{C}$ com perfuração e, também, FOP.

Em todos os casos, exceto um, o menor orifício era sempre póstero-inferior, mais próximo à veia cava inferior, e o maior, mais anterior e próximo à aorta. Curiosamente, era sempre o orifício menor o primeiro a ser cruzado.

O implante de duas próteses só não foi possível em um caso (FISD) em que se perdeu a posição da guia no orifício menor. Como não foi possível recruzar o defeito com facilidade, optou-se por deixá-lo aberto e fechar apenas o maior.
A Tabela 2 mostra a relação do número dos orifícios, a distância entre eles, os diâmetros dos orifícios e as próteses utilizadas. O diâmetro dos orifícios menores variou de $4 \mathrm{~mm}$ a $16 \mathrm{~mm}(10,5 \pm 4,0 \mathrm{~mm})$ e dos maiores, de $11 \mathrm{~mm}$ a $28 \mathrm{~mm}(20,6 \pm 5,5 \mathrm{~mm})$.

Foram utilizadas 24 próteses em 12 pacientes: 23 Amplatzer para CIA e uma Amplatzer para FOP.

$\mathrm{O}$ diâmetro central das próteses para $\mathrm{ClA}$ variou de $5 \mathrm{~mm}$ a $34 \mathrm{~mm}(16,8 \pm 7,6 \mathrm{~mm})$. A prótese para FOP utilizada foi de $25 \mathrm{~mm}$.

O tempo médio de seguimento foi de 19,8 meses e não houve complicações maiores nem óbitos.

Uma paciente, no final do exame, apresentou episódio de fibrilação atrial revertida por cardioversão elétrica, deixando o laboratório em ritmo sinusal. Outra paciente apresentou episódio de taquicardia atrial transitória, que reverteu espontaneamente, ainda na sala de exame.

Não houve embolizações imediatas ou tardias das próteses.

Os ecocardiogramas de controle imediato demonstraram shunt residual em dois $(16,6 \%)$ pacientes. Uma paciente (AMA) apresentou mínimo shunt residual, que desapareceu espontaneamente em três meses. Outro paciente (ESG), que apresentava pequeno shunt residual entre as duas próteses imediatamente após a liberação delas, mantém o shunt no seguimento ecocardiográfico de três meses, realizado recentemente. 

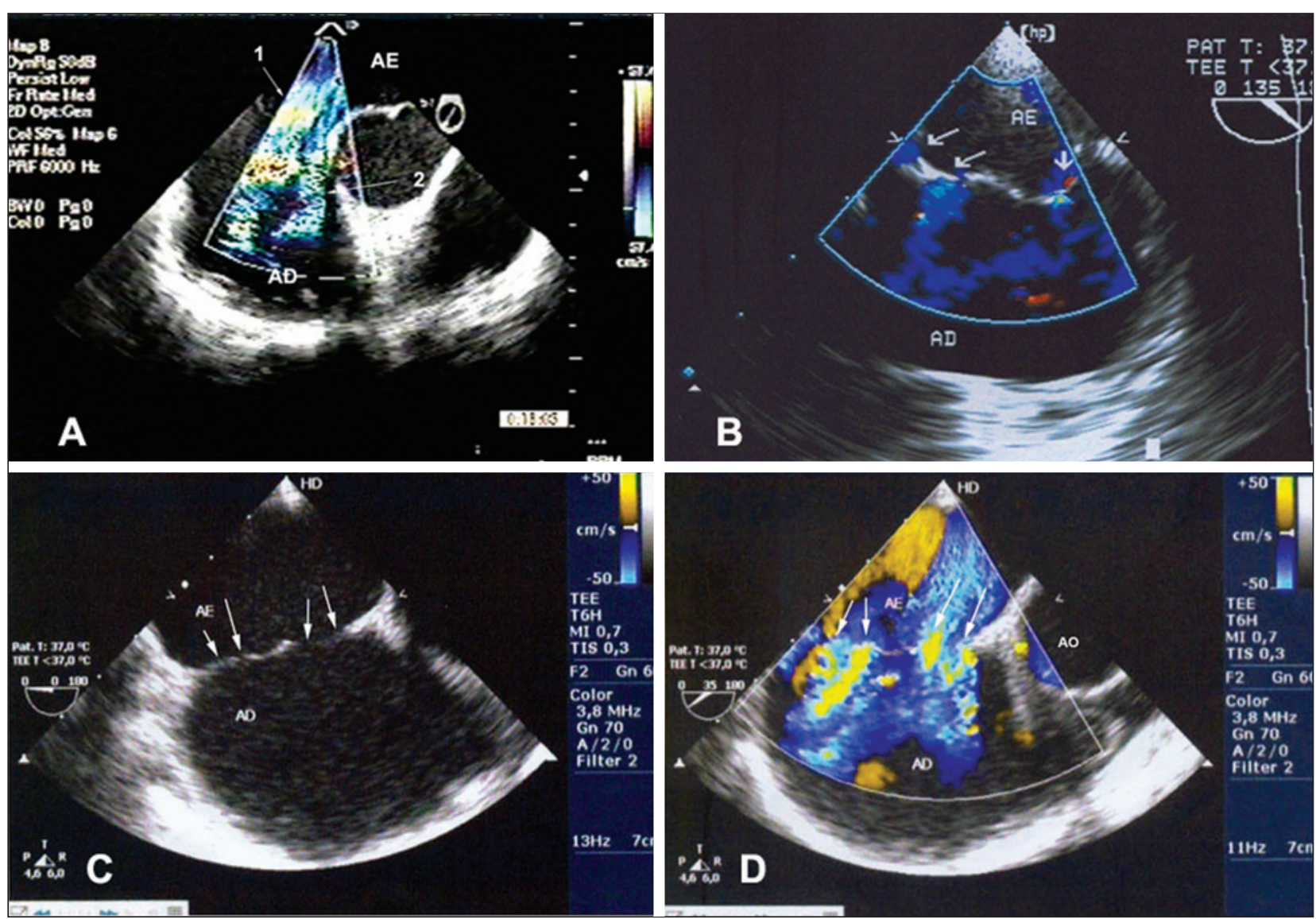

Figura 1 - No painel A, Doppler em cores demonstra dois orifícios contíguos passíveis de fechamento com uma única prótese. Em B, observamse três orifícios distintos, com o maior no centro (o implante de uma prótese junto à aorta e outra no orifício maior promoveu a oclusão completa dos três defeitos). Em C, a imagem bidimensional apenas sugere a presença dos quatro orifícios, mais bem identificados em D, por meio do fluxo em cores.

Vale ressaltar que o paciente FISD continua com fluxo pelo orifício menor, deixado aberto. Esse orifício tinha o diâmetro estático de $4 \mathrm{~mm}$, que passou a 13 $\mathrm{mm}$ depois de estirado. O orifício maior em que se implantou a prótese está completamente ocluído, sem shunt residual. O paciente se mantém assintomático após dois anos e aguarda o fechamento do segundo orifício com nova prótese.

Acrescentamos à casuística mais dois pacientes (GBV, SCS), que tinham tido suas comunicações fechadas há mais de um ano e que apresentaram shunts residuais significativos, necessitando de um segundo dispositivo para a oclusão completa dos defeitos.

A primeira paciente (GBV), uma criança de quatro anos à época do implante, tinha CIA de $9 \mathrm{~mm}$ ao ETE. O diâmetro estirado mediu $20 \mathrm{~mm}$. A CIA foi fechada com prótese Helex Septal Occluder de 30 mm (A L Gore and Associates - Fagstaff, Arizona, Estados Unidos). Apesar de a primeira prótese se manter em boa posição, mantinha shunt residual de $4 \mathrm{~mm}$, ao ETE, pela borda aórtica. Foi tomada a decisão de implantar nova prótese, o que foi feito três anos após. Foi utilizada ASO de $9 \mathrm{~mm}$, obtendo-se oclusão completa do defeito residual.

O outro caso (SCS), de 37 anos, paciente portadora de síndrome de Marfan, recusava terminantemente a cirurgia convencional. A avaliação do caso mostrou uma raiz aórtica de diâmetro normal e uma CIA medindo $33 \mathrm{~mm}$ ao ETE. O diâmetro estirado foi de $40 \mathrm{~mm}$. Teve implantada uma prótese ASO de $40 \mathrm{~mm}$ que demonstrou, no seguimento, shunt residual de $8 \mathrm{~mm}$ na borda da veia cava superior. Foi implantada nova prótese ASO $20 \mathrm{~mm}$ no pertuito residual, há 16 meses, com sucesso, estando o shunt completamente ocluído, conforme observado nos ecocardiogramas de controle.

É importante relatar que, no seguimento clínico médio de 18 meses, nenhum dos pacientes estudados apresentou arritmias.

\section{DISCUSSÃO}

O fechamento de CIA com próteses Amplatzer já é um procedimento consagrado, com alto índice de 
TABELA 2

Comunicações interatriais múltiplas - medida dos orifícios e próteses utilizadas

\begin{tabular}{|c|c|c|c|c|c|c|c|c|}
\hline$N$ & ID & $\begin{array}{l}\text { Número de } \\
\text { orifícios }\end{array}$ & $\begin{array}{c}\text { Distância } \\
(\mathbf{m m})\end{array}$ & $\begin{array}{c}\varnothing \text { maior } \\
(\mathrm{mm})\end{array}$ & Prótese & $\begin{array}{c}\varnothing \text { menor } \\
(\mathrm{mm})\end{array}$ & Prótese & Resultado \\
\hline 1 & FISD & 3 & 28 & 28 & ASO 34 & 10 & ASO 10(falha) & $\begin{array}{c}\text { Shunt moderado pelo } \\
\text { orifício menor }\end{array}$ \\
\hline 2 & FCC & 2 & 16 & 21 & ASO 22 & 11 & ASO 12 & Sem shunt \\
\hline 3 & AMA & 4 & 20 & 24 & ASO 26 & 4 & ASO 5 & Mínimo shunt \\
\hline 4 & SAS & 2 & 12 & 28 & ASO 28 & 10 & ASO 10 & Sem shunt \\
\hline 5 & DFSC & 2 & 10 & 27 & ASO 28 & 10 & ASO 12 & Sem shunt \\
\hline 6 & SDM & 2 & 14 & 21 & ASO 24 & 8 & ASO 12 & Sem shunt \\
\hline 7 & AMM & 4 & 10 & 17 & ASO 18 & 9 & ASO 10 & Sem shunt \\
\hline 8 & MAS & 2 & 21 & 14 & ASO 14 & 10 & ASO 11 & Sem shunt \\
\hline 9 & MCMW & 2 & 14 & 16 & ASO 17 & 15 & ASO 15 & Sem shunt \\
\hline 10 & COW & $\mathrm{FOP}+\mathrm{ClA}$ & 13 & 11 & PFO 25 & 4 & ASO 12 & Sem shunt \\
\hline 11 & ALS & 5 & 13 & 25 & ASO26 & 16 & ASO 16 & Sem shunt \\
\hline 12 & ESG & 4 & 10 & 20 & ASO 24 & 6 & ASO 6 & Pequeno shunt \\
\hline 13 & $\mathrm{GBV}^{*}$ & Shunt residual & 0 & 8,5 & ASO 9 & - & - & Sem shunt \\
\hline 14 & SCS* & Shunt residual & 0 & 18 & ASO 20 & - & - & Sem shunt \\
\hline
\end{tabular}

* Pacientes com shunt residual.

$\mathrm{ID}=$ identificação do paciente; FOP = forame oval patente; CIA = comunicação interatrial.

sucesso e segurança, mesmo em crianças $^{10}$. Apesar disso, ainda há poucos estudos sobre o uso de mais de uma prótese, principalmente relatando o seguimento a longo prazo ${ }^{11-15}$.

Nosso objetivo é relatar a experiência com o uso de duas próteses implantadas simultaneamente, ou não, e discutir os aspectos técnicos e o seguimento desses pacientes.

Embora, na maioria dos casos, o ETE realizado por vários ecocardiografistas diferentes já houvesse previsto a possibilidade do uso de duas próteses, em dois casos não foi capaz de perceber os defeitos adicionais durante o procedimento. A relativa inexperiência do ecocardiografista com esse tipo de defeito em um caso e a posição do orifício adicional em outro podem ter sido as possíveis causas. No fechamento das CIAs, o ETE é de fundamental importância, desde a indicação do procedimento até a obtenção das imagens adequadas ao fechamento. É vital que o ecocardiografista seja familiarizado com os defeitos congênitos do coração, além de possuir a destreza no manuseio da sonda transesofágica. Se esses dois pré-requisitos não forem atendidos, o resultado final ficará comprometido. Na maioria das vezes, será feito o diagnóstico da CIA, mas não serão fornecidos detalhes importantes, como a presença e o tipo das bordas do defeito, bem como a presença de defeitos adicionais.

Tem sido um problema a identificação dos orifícios adicionais, principalmente quando em posição póstero-inferior, numa região mal visualizada pelo ETE.
Essa talvez seja uma das melhores indicações do uso do ecocardiograma intracardíaco (EIC), que, sob esse ponto de vista, é superior ao ETE.

Quando não percebidos previamente pelo ETE, os defeitos adicionais são reconhecidos porque, geralmente, são os primeiros a serem cruzados com a guia hidrofílica ou o cateter diagnóstico. Chama a atenção, nesses casos, ver o cateter cruzar o septo muito abaixo do orifício alvo. Uma das vezes, só fomos ter a certeza de estar no orifício errado depois de insuflar o balão medidor e verificar que ele ficava longe da aorta.

Nessa situação, é fundamental o papel do ecocardiograma, uma vez que só ele é capaz de determinar em que ponto do septo está o cateter ou a guia. A melhor solução, nesses casos, é, mantendo a guia no defeito menor, tentar cruzar com uma segunda guia pelo orifício desejado. Se não for alcançado esse objetivo, oclui-se o defeito menor pela insuflação de um balão medidor de $24 \mathrm{~mm}$.

Uma vez tendo cruzado o orifício desejado, medese seu diâmetro estirado e implanta-se a prótese para, só depois de ter a certeza do desaparecimento do shunt, retirar a guia do defeito menor.

Muitas vezes, uma única prótese pode ser capaz de ocluir mais de um defeito. Isso ocorre quando há confluência dos defeitos contíguos, criando um defeito único, seja por meio de atriosseptostomia por balão ${ }^{16}$ seja pela simples insuflação do balão medidor, o que, naturalmente, depende da rigidez das bordas dos 
orifícios $^{8}$. Por não ser desprovida de riscos, a atriosseptostomia não nos parece uma boa idéia e nunca a utilizamos nesse contexto.

Quando os orifícios distam até $7 \mathrm{~mm}$ a $8 \mathrm{~mm}$ entre a borda de um orifício e a borda distal do outro, também é possível o uso de uma única prótese $^{4,17}$. Nesse caso, busca-se implantar o dispositivo no orifício maior, com o objetivo de cobrir também o menor, obtendo taxas de oclusão imediata bastante satisfatórias ${ }^{18}$.

Nos casos em que há necessidade de duas próteses, idealmente deverá ser tentado o implante simultâneo dos dois dispositivos: inicialmente, o dispositivo menor, para, em seguida, posicionar o maior por cima do primeiro, de forma a fazer com que seus discos o envolvam (Figura 2).

Algumas vezes, os orifícios são tão distantes entre si ou em planos espaciais tão diversos que o alinhamento das próteses é impossível. Nesse caso, é importantíssimo que os dois dispositivos sejam cuidadosamente avaliados quanto a seu tamanho e relação com as estruturas vizinhas. Em nossos casos não houve, até o momento, qualquer tipo de problema com essa situação (Figura 3).

Ocasionalmente, o implante simultâneo não é possível, às vezes porque só se percebeu o orifício adicional após a oclusão do maior, outras porque se acreditou que apenas um dispositivo seria capaz de ocluir os dois defeitos. Nesses casos, cruzar o orifício menor pode ser tecnicamente difícil e apenas com o direcionamento pelo ETE e o uso de guias hidrofílicas o

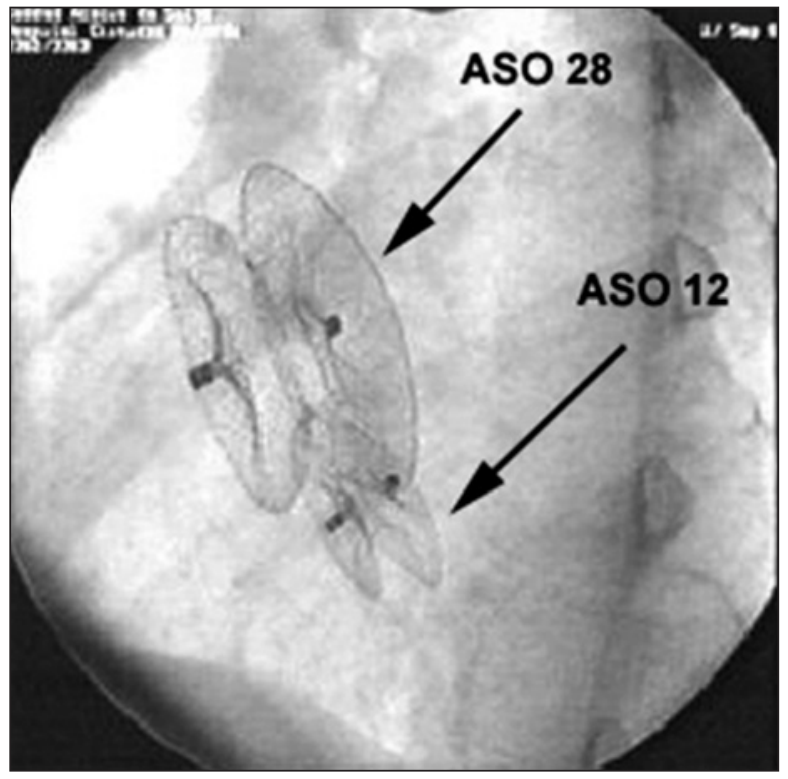

Figura 2 - Implante simultâneo de duas próteses, em que se observa a maior (ASO 28) envolvendo a menor (ASO 12). Ambos os dispositivos estão alinhados corretamente. objetivo é alcançado. A colocação da segunda prótese costuma ser fácil, ficando, em geral, bastante afastada da primeira prótese.

O conceito de que o uso de dois dispositivos, principalmente quando os discos de metal se superpõem, impediria a endotelização completa ${ }^{19}$ parece não se sustentar hoje em dia ${ }^{11}$.

Optamos por incluir os dois casos de shunt residual porque o objetivo deste trabalho é avaliar as dificuldades e as conseqüências do implante de duas próteses, simultânea ou seqüencialmente.

Nos casos em que houve shunt residual após o implante do primeiro dispositivo, normalmente aguardamos o fechamento espontâneo por pelo menos um ano. Se a oclusão completa do defeito não ocorrer, procedemos ao implante da segunda prótese.

Nesse tipo de procedimento, a maior dificuldade é localizar e cruzar o pertuito, sem interferir com a primeira prótese. O uso de guia hidrofílica é de vital importância nesses casos.

Em ambos os casos, utilizamos próteses Amplatzer, pela sua facilidade de uso e maior maleabilidade.

No primeiro caso (CBV), o pertuito tinha a forma de um túnel longo, que começava na borda aórtica do defeito e se abria na periferia do disco direito no átrio direito. Ao implantar a segunda prótese, procuramos introduzir o disco esquerdo e a porção central, completamente dentro do pertuito, deixando aberto apenas o disco direito. Dessa forma, conseguimos ocupar completamente o espaço e fechar totalmente o shunt residual (Figura 4).

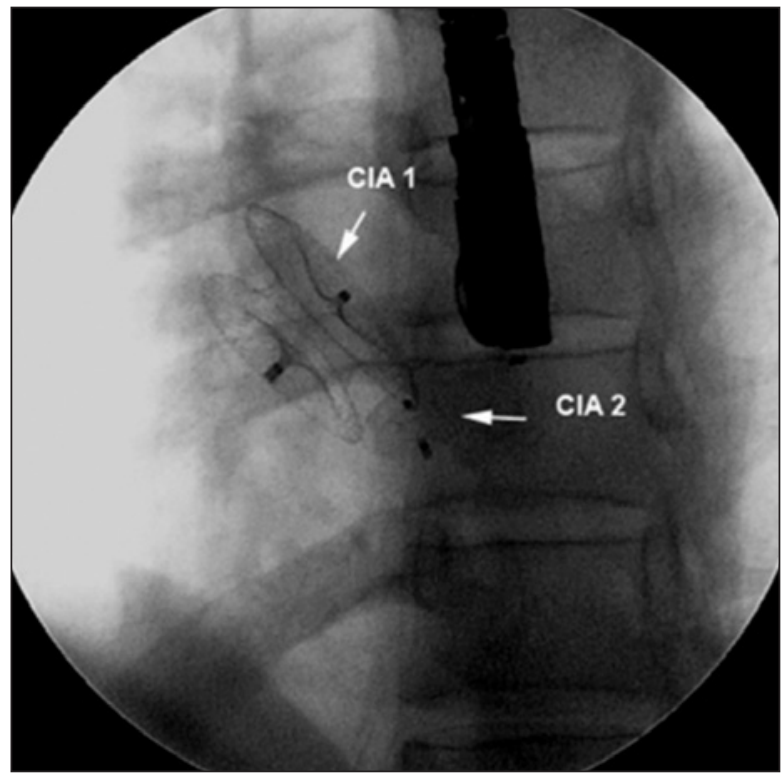

Figura 3 - Implante de duas próteses, afastadas uma da outra. Observam-se os planos diferentes em que estão as duas próteses, quase em ângulo reto. 
Chamié F, et al. Oclusão Percutânea dos Defeitos do Septo Atrial Utilizando Mais de Uma Prótese. Rev Bras Cardiol Invas. 2008;16(1):77-85.

No segundo caso (SCS), implantamos a segunda prótese da maneira habitual (Figura 5).

Comparando-se as duas situações, achamos que o implante de uma segunda prótese em orifício residual, com o primeiro dispositivo já endotelizado, é mais fácil que o implante simultâneo, uma vez que o procedimento se torna muito parecido com o implante de uma única prótese.

\section{CONCLUSÕES}

Apesar de adicionar algum grau de dificuldade ao
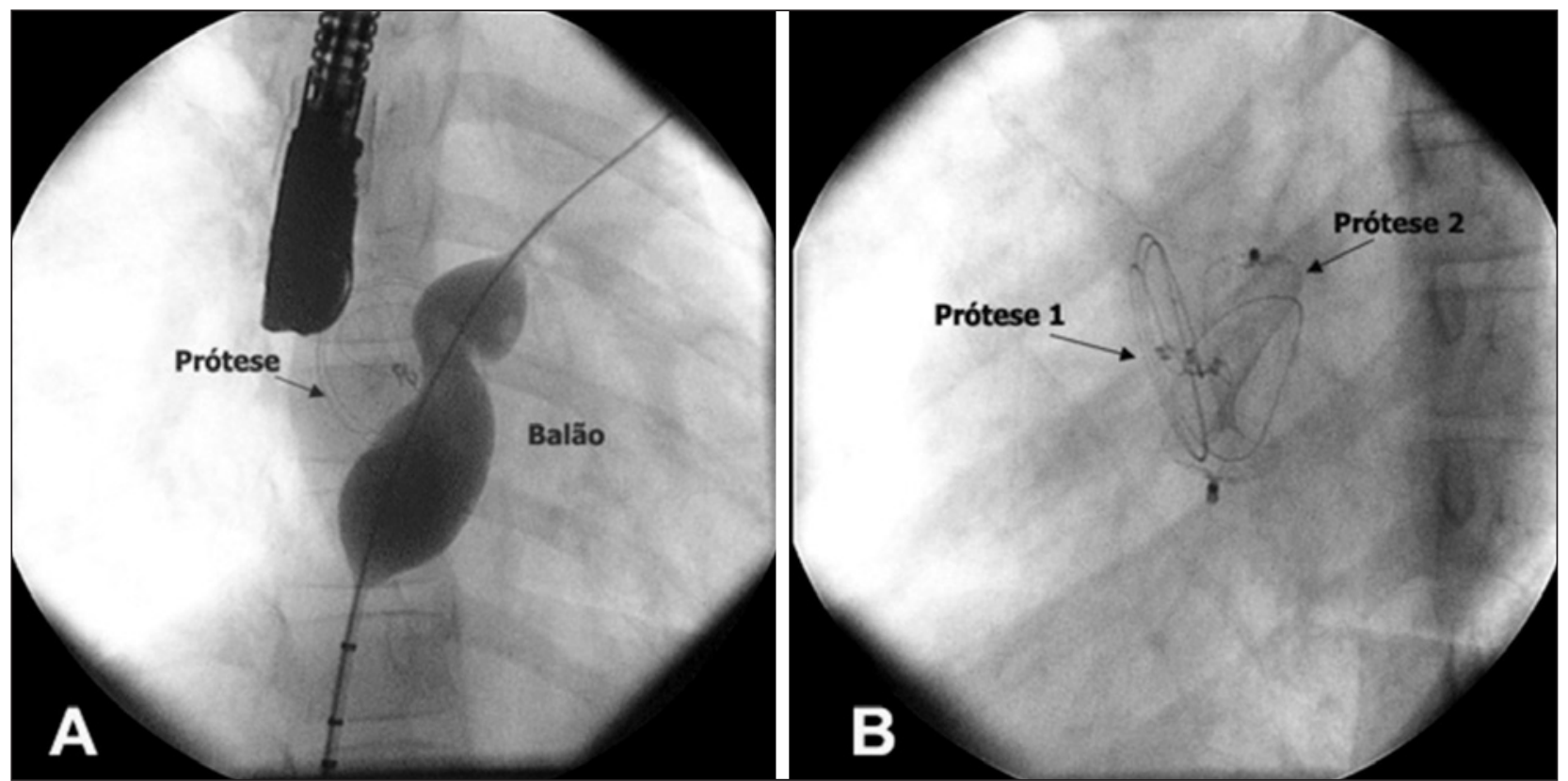

Figura 4 - No painel A, observa-se a primeira prótese implantada e o pertuito, cruzado pelo balão de oclusão sobre corda-guia introduzida em veia pulmonar superior esquerda. Observa-se a forma do pertuito, em túnel longo, delineada pelo balão. Em B, a segunda prótese já implantada. Nota-se que o disco esquerdo, deformado, ocupa a totalidade do pertuito, ocluindo-o completamente.
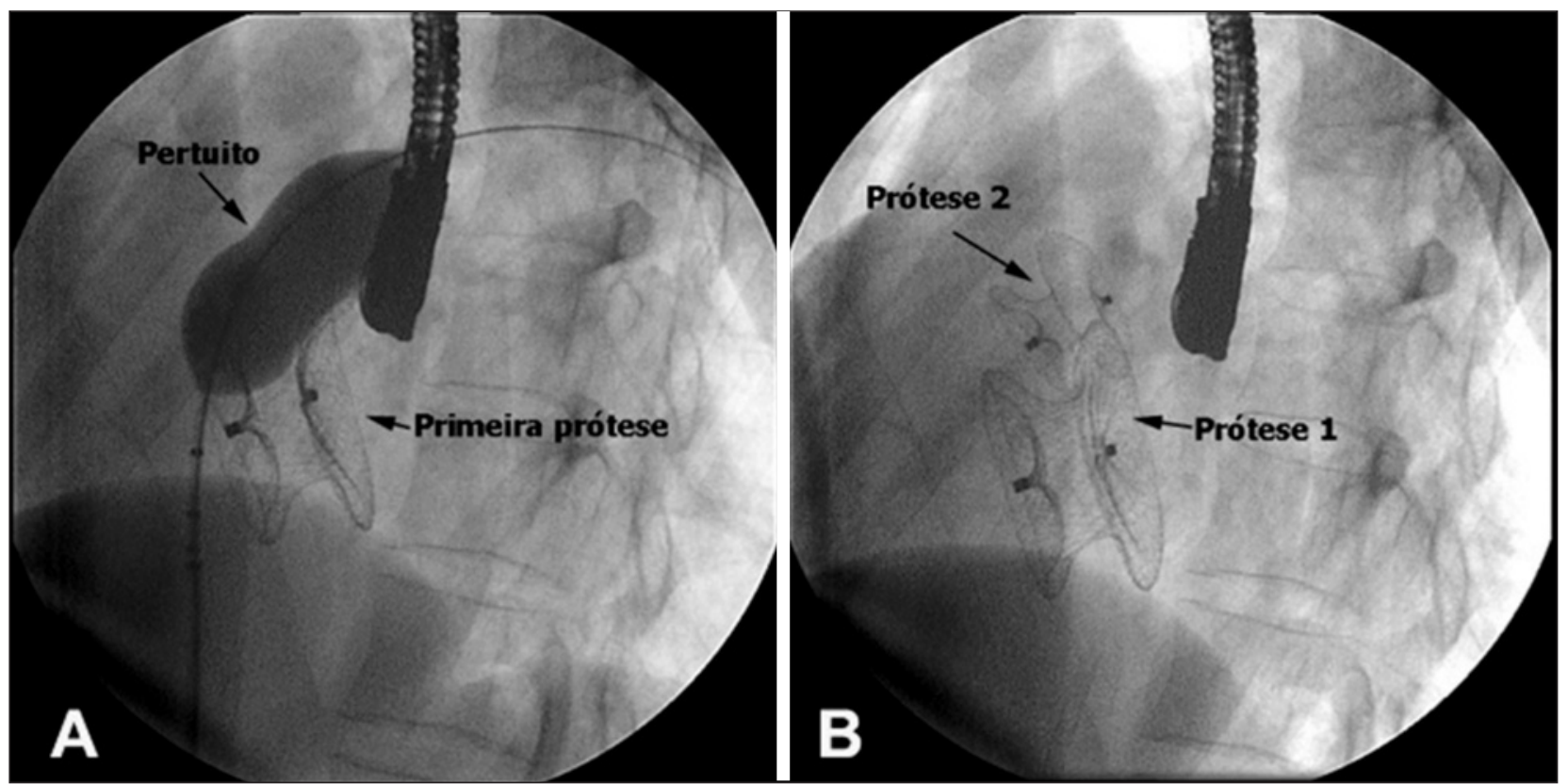

Figura 5 - No painel A, observa-se a medida do diâmetro estirado do shunt residual pelo balão medidor. Em B, a segunda prótese implantada, em posição mais póstero-superior, ao lado da primeira. 
procedimento, o fechamento de defeitos múltiplos com mais de uma prótese é factível, seguro e eficaz.

O ETE é fundamental na indicação e no manuseio desse tipo de paciente.

O EIC apresenta pequena vantagem sobre o ETE, na medida em que mostra melhor a porção inferior do septo interatrial, local onde incide a maioria dos pequenos defeitos adicionais.

Embora outros tipos de próteses venham sendo utilizadas nesse contexto, achamos que as próteses Amplatzer são as que melhor se prestam a esse tipo de fechamento, pela grande disponibilidade de tamanhos, pela sua facilidade de uso e pela maior adaptabilidade a tipos diferentes de anatomia.

Até o momento, o uso de mais de um dispositivo metálico não parece causar nenhum dano ao septo atrial ou a estruturas nobres circunvizinhas, além de apresentar poucas complicações maiores; entretanto, mais estudos ainda se fazem necessários para definir a segurança desse procedimento, a longo prazo, especialmente em crianças.

\section{REFERÊNCIAS BIBLIOGRÁFICAS}

1. Podnar T, Martanovic P, Gavora P, Masura J. Morphological variations of secundum-type atrial septal defects: feasibility for percutaneous closure using Amplatzer septal occluders. Catheter Cardiovasc Interv. 2001;53(3):386-91.

2. Ewert $\mathrm{P}$, Berger F, Vogel M, Dähnert I, Alexi-Meshkishvili $\mathrm{V}$, Lange PE. Morphology of perforated atrial septal aneurysm suitable for closure by transcatheter device placement. Heart. 2000;84(3):327-31.

3. Bartz PJ, Cetta F, Cabalka AK, Reeder GS, Squarcia U, Agnetti A, et al. Paradoxical emboli in children and young adults: role of atrial septal defect and patent foramen ovale device closure. Mayo Clin Proc. 2006;81(5):615-8.

4. Cao Q, Radtke W, Berger F, Zhu W, Hljazi ZM. Transcatheter closure of multiple atrial septal defects. Initial results and value of two- and three-dimensional transoesophageal echocardiography. Eur Heart J. 2000;21(11):941-7.

5. Masura J, Gavora P, Formanek A, Hijazi ZM. Transcatheter closure of secundum atrial septal defects using the new self-centering Amplatzer septal occluder: initial human experience. Cathet Cardiovasc Diagn. 1997;42(4):388-93.

6. Han YM, Gu X, Titus JL, Rickers C, Bass JL, Urness M, et al. New self-expanding patent foramen ovale occlusion device. Cathet Cardiovasc Interv. 1999;47(3):370-6.

7. Mügge A, Daniel WG, Angermann C, Spes C, Khandheria
BK, Kronzon I, et al. Atrial septal aneurysm in adult patients. A multicenter study using transthoracic and transesophageal echocardiography. Circulation. 1995;91(11):2785-92.

8. Chamié F, Chamié D, Ramos S, Tress JC, Victer R. Fechamento percutâneo das comunicações interatriais $(\mathrm{CIA})$ complexas. Rev Bras Cardiol Invas. 2006;14(1):47-55.

9. Boutin C, Musewe NN, Smallhorn JF, Dyck JD, Kobayashi T, Benson LN. Echocardiographic follow-up of atrial septal defect after catheter closure by double-umbrella device. Circulation. 1993;88(2):621-7.

10. Du ZD, Hijazi ZM, Kleinman CS, Silverman NH, Larntz K, Amplatzer investigators. Comparison between transcatheter and surgical closure of secundum atrial septal defect in children and adults: results of a multicenter nonrandomized trial. J Am Coll Cardiol. 2002;39(11):1836-44.

11. Awad SM, Garay FF, Cao QL, Hijazi ZM. Multiple Amplatzer septal occluder devices for multiple atrial communications: immediate and long-term follow-up results. Catheter Cardiovasc Interv. 2007;70(2):265-73.

12. Pinto RJ, Dalvi B. Closure of two atrial septal defects using two separate Amplatzer ASD devices. Indian Heart J. 2005;57(3):251-4.

13. Pedra CA, Pedra SR, Esteves CA, Cassar R, Pontes SC Jr, Braga SL, et al. Transcatheter closure of secundum atrial septal defects with complex anatomy. J Invasive Cardiol. 2004;16(3):117-22.

14. Dobrolet N, Iskowitz S, Lopez L, Whalen R, Zahn E. Sequential implantation of two Helex septal occluder devices in a patient with complex atrial septal anatomy. Catheter Cardiovasc Interv. 2001;54(2):242-6.

15. Suárez De Lezo J, Medina A, Pan M, Romero $M$, Segura J, Pavlovic D, et al. Transcatheter occlusion of complex atrial septal defects. Catheter Cardiovasc Interv. 2000; 51(1):33-41

16. Carano N, Hagler DJ, Agnetti A, Squarcia U. Device closure of fenestrated atrial septal defects: use of a single Amplatz atrial septal occluder after balloon atrial septostomy to create a single defect. Catheter Cardiovasc Interv. 2001;52(2):203-7.

17. Pedra CA, Fontes-Pedra SR, Esteves CA, Assef J, Fontes VF, Hijazi ZM. Multiple atrial septal defects and patent ducts arteriosus: successfull outcome using two Amplatzer septal occluders and Gianturco coils. Cathet Cardiovasc Diagn. 1998;45(3):257-9.

18. Szkutnik M, Masura J, Bialkowski J, Gavora P, Banaszak P, Kusa J, et al. Transcatheter closure of double atrial septal defects with a single Amplatzer device. Catheter Cardiovasc Interv. 2004;61(2):237-41.

19. Zanchetta M, Rigatelli G, Pedon L, Zennaro M, Carrozza A, Onorato E. Catheter closure of perforated secundum atrial septal defect under intracardiac echocardiographic guidance using a single Amplatzer device: feasibility of a new method. J Invasive Cardiol. 2005;17(5):262-5. 